\title{
Gallbladder agenesis and atrial septal defect: A case report
}

\author{
YONGQIANG LIU $^{1 *}$, NI AO ${ }^{2 *}$ and HAIPING ZHAO ${ }^{1}$ \\ ${ }^{1}$ Department of General Surgery, The Affiliated Hospital of Inner Mongolian Medical University, Hohhot, \\ Inner Mongolia Autonomous Region 010059; ${ }^{2}$ Inner Mongolia International Mongolian Hospital, \\ Hohhot, Inner Mongolia Autonomous Region 010010, P.R. China
}

Received December 22, 2014; Accepted August 11, 2015

DOI: $10.3892 / \mathrm{etm} .2016 .3115$

\begin{abstract}
Congenital absence of gallbladder and atrial septal defect (ASD) are clinically rare congenital organ malformations, and the simultaneous occurrence of the two is even more rare. The present study reported a case of gallbladder agenesis combined with congenital ASD. A 38-year-old male patient presented with a 3-year history of recurring upper right abdominal pain. The pain had no evident cause and was accompanied by dyspepsia and gasteremphraxis with indigestion. Several color Doppler ultrasonography scans revealed cholecystitis and gallbladder stones. A physical examination revealed cardiac murmur. A color Doppler ultrasonography of the heart was indicative of congenital heart disease. A corrective surgery for ASD was performed. Subsequently, a mini-incision cholecystectomy was performed as explorative surgery. A magnetic resonance cholangiopancreatography scan of the abdomen was performed in order to confirm the diagnosis of gallbladder disorder and cystic duct hypoplasia. A final definite diagnosis of gallbladder agenesis was confirmed. In conclusion, atrophy and gallbladder full of stones are frequently misdiagnosed, and establishing a definitive preoperative diagnosis is difficult. Awareness of this congenital malformation may assist physicians in distinguishing cases with unclear manifestation and avoiding unnecessary surgical interventions.
\end{abstract}

\section{Introduction}

Concurrent manifestation of congenital absence of gallbladder and atrial septal defect (ASD) is a rare congenital organ malformation in clinical practice (1). No apparent association has been identified between these two congenital organ

Correspondence to: Dr Haiping Zhao, Department of General Surgery, The Affiliated Hospital of Inner Mongolian Medical University, 1 North Tongdao Street, Hohhot, Inner Mongolia Autonomous Region 010059, P.R. China

E-mail: zhaohaiping007@yahoo.com.cn

${ }^{*}$ Contributed equally

Key words: congenital, cholecyst, atrial septal defect, agenesis malformations. Sufficient attention must be paid to these disorders as they are often misdiagnosed or overlooked during diagnosis. The incidence of gallbladder agenesis in the general population is reported to be $13-65 / 100,000$ (2). In clinical practice, the incidence is $0.007-0.0027 \%$, while in autopsy series it is $0.04-0.13 \%$ (3). Females are more commonly affected (ratio, $3: 1$ ), and $>50 \%$ of patients with gallbladder agenesis are symptomatic and require surgical intervention $(4,5)$. It is crucial that clinicians consider gallbladder agenesis in cases where the gallbladder appears abnormal on preoperative imaging studies and cannot be identified using laparoscopy (6). Preoperative diagnostic imaging such asmagnetic resonance cholangiopancreatography (MRCP) and endoscopic ultrasound should be considered. If such a condition is encountered during surgery, intraoperative cholangiography and intraoperative ultrasound may be performed to rule-out agenesis and ectopic gallbladder (7). In the present case report, the diagnosis and surgical intervention of gallbladder agenesis and ASD is described.

\section{Case report}

A 38-year-old male visited the Affiliated Hospital of Inner Mongolia Medical University (Hohhot, China) in December 2010 with a 3-year history of recurring upper right abdominal pain, which had deteriorated in the last week. The pain had no evident cause. It was accompanied by dyspepsia and gasteremphraxis with indigestion, and was exacerbated following meals.

Several color Doppler ultrasonography scans were performed in our hospital, which revealed cholecystitis and gallbladder stones. In the week prior to hospital admission, the aforementioned symptoms deteriorated and thus the patient visited our hospital for treatment. Ultrasound imaging had been previously performed in the Inner Mongolia Xilingol League Hospital (Xilingol League, China), and further scans were subsequently conducted on patient admission to our hospital. Following physical examination, cardiac murmur was detected. In addition, the color Doppler ultrasonography scans of the heart were indicative of congenital heart disease (CHD). The patient was admitted to the Cardiovascular Surgery Department of our hospital under the diagnosis of CHD with concomitant congenital ASD and chronic atrophic cholecystitis with gallbladder stones. During the 34 days of hospitalization, the patient exhibited occasional palpitations and shortness of breath, fatigue and cyanosis of the lips. 


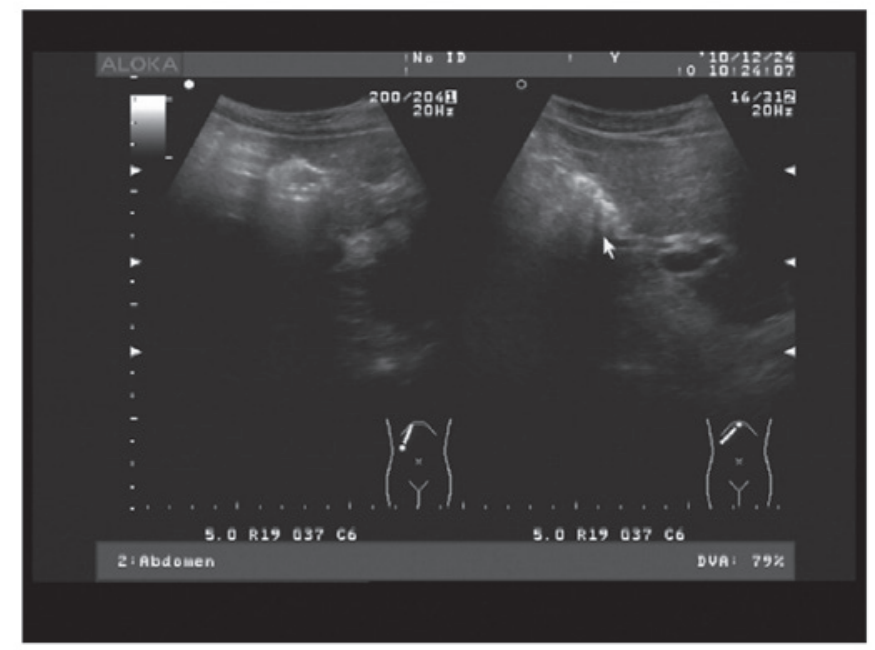

Figure 1. Ultrasound showing the absence of bile echo in the gallbladder in visible outline, gallbladder contraction, and high echo.

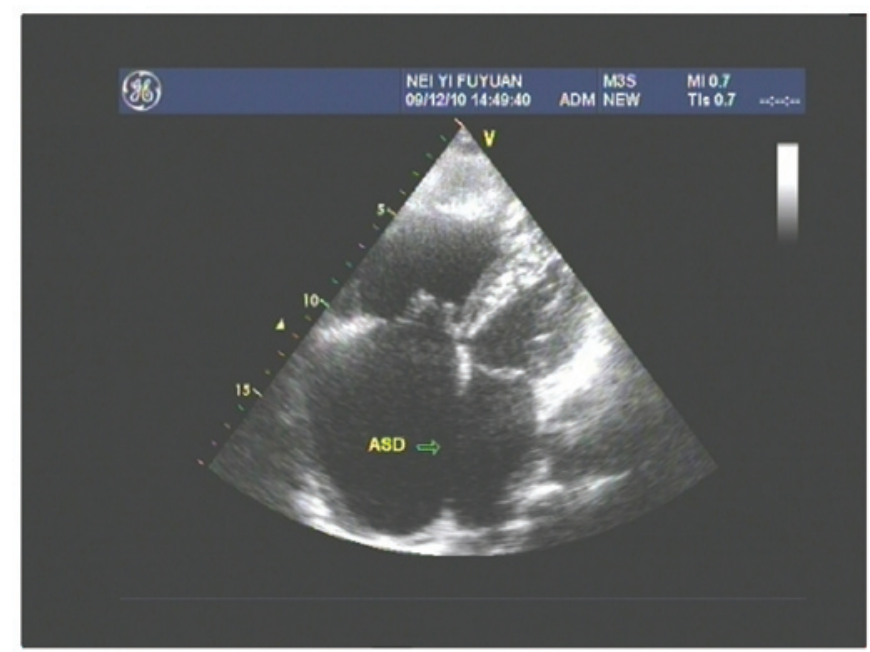

Figure 2. Ultrasound showing the ASD. ASD, atrial septal defect.

The medical history of the patient included self-reported palpitations and shortness of breath following exercise. In addition, the patient had been prone to activity-induced fatigue since childhood, and had a history of susceptibility to respiratory tract infection.

Auscultation of the pulmonary valve revealed accentuation of the second heart sound at the left side of the sternum, between the first and second ribs, and grade $3 / 6$ systolic murmur. Furthermore, a soft abdomen without upper right abdominal pain on palpation, rebound tenderness, muscle rigidity, a negative Murphy's sign and borborygmus at a rate of $3 /$ min were identified.

Upon auxiliary examination, color Doppler ultrasound of the abdomen indicated chronic atrophic cholecystitis with gallbladder stones. Fig. 1 shows a color Doppler ultrasound of the congenital ASD, atrial septum consistency degradation, echo loss of $50 \mathrm{~mm}$, large left-to-right shunt volume and a flow rate of $0.73 \mathrm{~m} / \mathrm{sec}$. Figs 2 and 3 show the left anterior descending artery examination results, which identified that the patient was positive for Hepatitis C virus antibody,

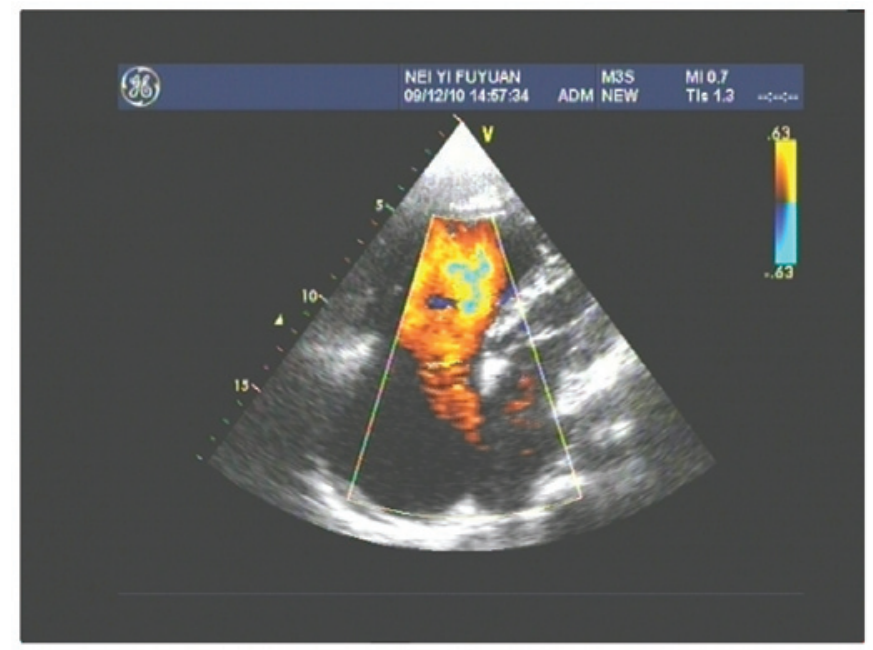

Figure 3. Ultrasound showing the direction of blood flow in the atrial septal defect. Red indicates left-to-right blood flow.

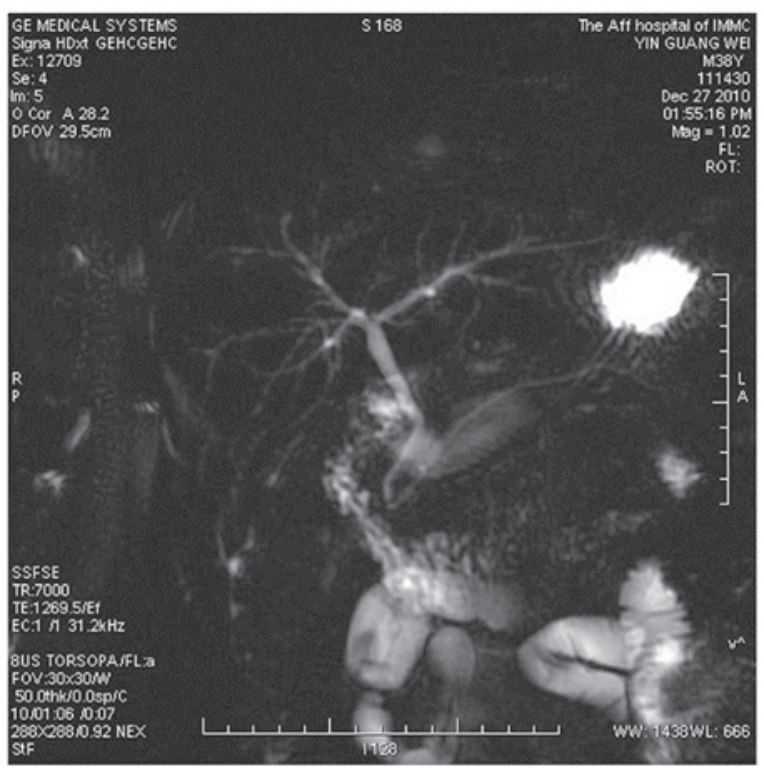

Figure 4. Magnetic resonance cholangiopancreatography showing the cystic duct and gallbladder agenesis.

while the results of routine blood test and biochemistry were normal.

Cardiovascular surgery was performed via a chest incision, and inter-ASD repair was achieved with extracorporeal circulation. At 12 days postoperatively, the patient recovered. Subsequently, a mini-incision cholecystectomy was performed to confirm the diagnosis of gallbladder agenesis. During surgical exploration, $\sim 8 \times 3 \times 2-\mathrm{mm}$ soft tissue fragments were found in the porta hepatis. During the dissection, normal, soft, fat-like tissue was observed, with no tissue changes in the chorionic and muscular layer, mucous membrane degradation or changes in the gallbladder tissue identified. Gallbladder agenesis was considered as a possible diagnosis during surgery, excluding the possibility of an outer-ectopic gallbladder. After gradually extending the incision site and freely dissecting the first part of the choledochus at a length of $\sim 9 \mathrm{~cm}$, the gallbladder, cystic duct and biliary branches were not found. The 
porta hepatis was dissociated from the liver, but the portal vein and tube wall were found intact.

Despite the dissociation of the porta hepatis from the liver, the gallbladder and cystic duct were still not found. Following discussion during the surgery, the surgical exploration was terminated and the inner liver, posterior pancreatic head and duodenum were examined using color Doppler ultrasonography. A magnetic resonance cholangiopancreatography (MRCP) scan of the abdomen was performed in order to confirm the diagnosis of gallbladder and cystic duct hypoplasia (Fig. 4). The patient recovered and was discharged from the hospital 5 days later, in January 2011. Following surgery, the patient received 6 phone follow-up consultations (once per month). The date of final follow-up was August 2015. No new symptoms, further treatment or outcomes occurred. No upper-right abdominal pain or surgical complications were reported. Written informed consent was obtained from the patient.

\section{Discussion}

Gallbladder agenesis is a rare gallbladder malformation caused by the agenesis of the liver diverticulum tail during the fourth week of the embryonic period $(8,9)$. Lemery and Bergman first reported this condition in 1701 (10). The incidence rate of gallbladder agenesis in countries other than China has been found to be $0.013-0.065 \%$ (11). The present case of gallbladder agenesis was confirmed during a surgical intervention in the patient during his initial visit to our hospital. Following the retrieval of hospital records, 33,983 cases of gallbladder removal were identified between 1975 and 2013, which translates to an incidence rate of $0.0029 \%$, with a rare prevalence in patients with gallbladder agenesis accompanied by ASD, which is below the reported rate for countries other than China. Singh et al (12) classified this condition into the following 3 types, according to their clinical features (13): i) Multiple congenital anomaly (12.8\%); ii) asymptomatic (31.6\%); and iii) symptomatic (55.6\%). The patient of the current study presented with a combination of a large ASD and type I congenital gallbladder agenesis.

Ultrasonography is the preferred imaging modality for gallbladder diseases. Due to the scarcity of reports on gallbladder agenesis and the different experiences among physicians, various confounding diagnoses of gallbladder agenesis exist in the clinical field. With regard to patients whose preoperative color Doppler ultrasounds show numerous calculi in the gallbladder, and gallbladder contraction, imaging practitioners and surgeons should be aware of this disease. Furthermore, patients with physical deformities in one organ should be examined for possible deformities in other organs. Patients with suspicion of gallbladder agenesis should undergo MRCP or endoscopic retrograde cholangiopancreatography (4) in order to confirm the diagnosis.

Surgical intervention is not recommended for patients who are suspected of having gallbladder agenesis but have exhibited no acute abdominal symptoms (14). Ectopic gallbladder should be excluded prior to or during surgery in order to confirm the diagnosis of gallbladder agenesis. The use of B-mode ultrasonography should be considered for perioperative diagnosis. Perioperative diagnosis can avoid the limitation of preoperative percutaneous B-mode ultrasonography and excessive and blind examination, in order to prevent an increased risk of iatrogenic damages. With regard to patients who exhibit local expansion of the hepatoduodenal ligament during surgery and those highly suspected of having gallbladder contraction near the common hepatic duct and choledochus, adequate deboning of the porta hepatis and the adhesion between the liver surface and transverse colon should be considered. Along the superior border of the duodenum, the choledochus to porta hepatis should be dissected, the full length of the outer-liver bile duct should be identified and the situation of gallbladder requires confirmation. The diagnosis of gallbladder agenesis should be definitive and only established after extensive examinations $(6,14)$.

Previous studies have demonstrated that a definitive diagnosis of gallbladder agenesis is challenging through ordinary preoperative examination $(4,15)$. MRCP is a noninvasive and effective method of biliary tract imaging, and it is not affected by cholestasis. It can also be used for exclusion of the diagnosis of ectopic gallbladder. In the present case, the patient was examined using MRCP following surgery. MRCP showed a good biliary tree image development, without observation of the gallbladder or ectopic gallbladder. Although MRCP cannot replace ultrasonography as a diagnostic modality for gallbladder diseases, it can provide anatomical information on the gallbladder fossa and has a clear advantage in the diagnoses of ectopic gallbladder and gallbladder agenesis (16). If the patient of the present case had been correctly diagnosed preoperatively, no unnecessary surgical exploration with a risk of complications would be required.

In conclusion, the present case attempted to provide an improved understanding of gallbladder agenesis, which would assist diagnosis, as well as the knowledge obtained by various examination methods. Thus, the diagnosis established should be as definitive as possible in order to avoid misdiagnosis. Physicians should be particularly aware of the fact that gallbladder agenesis tends to be discovered and diagnosed during surgical removal of the gallbladder. Due to the lack of insight in gallbladder agenesis, surgeons often conduct an extensive search for the gallbladder and dissect the bile duct, porta hepatis tissue duodenum and other parts, which can damage the biliary tract and cause unnecessary injuries.

\section{Acknowledgements}

This study was supported by a grant from the Ministry of Health of P.R. China (grant no. 201302073).

\section{References}

1. Langer T, Baudenbacher R, Hablützel K and Kehl O: Gallbladder agenesis: A diagnostic problem? Schweiz Rundsch Med Prax 30: 646-648, 1989 (In German).

2. Richards RJ, Taubin H and Wasson D: Agenesis of the gallbladder in symptomatic adults. A case and review of the literature. J Clin Gastroenterol 16: 231-233, 1993.

3. Peloponissios N, Gillet M, Cavin R and Halkic N: Agenesis of the gallbladder: A dangerously misdiagnosed malformation. World J Gastroenterol 11: 6228-6231, 2005.

4. Singh BG, Rao KPK, Ghosh SR and Chaudhry R: Congenital absence of gall bladder. Med J Armed Forces India 59: 152-153, 2003. 
5. Kasi PM, Ramirez R, Rogal SS, Littleton K and Fasanella KE: Gallbladder agenesis. Case Rep Gastroenterol 5: 654-662, 2011.

6. Stephenson JA, Norwood M, Al-Leswas D, Al-Taan O, Beable R, Lloyd DM and Dennison AR: Hepatic haemangioma masquerading as the gallbladder in a case of gallbladder agenesis: A case report and literature review. HPB Surg 2010: 971609, 2010.

7. Wilson JE and Deitrick JE: Agenesis of the gallbladder: Case report and familial investigation. Surgery 99: 106-109, 1986.

8. Haddock G, Morran CG and Anderson JR: Agenesis of the gallbladder. J R Coll Surg Edinb 31: 100-101, 1986.

9. Gotohda N, Itano S, Horiki S, Endo A, Nakao A, Terada N and Tanaka N: Gallbladder agenesis with no other biliary tract abnormality: Report of a case and review of the literature. J Hepatobiliary Pancreat Surg 7: 327-330, 2000.

10. Nadeau LA, Cloutier WA, Konecki JT, Morin G and Taylor RW: Hereditary gallbladder agenesis: Twelve cases in the same family. J Maine Med Assoc 63: 1-4, 1972.
11. Malde S: Gallbladder agenesis diagnosed intra-operatively: A case report. J Med Case Rep 4: 285, 2010.

12. Singh B, Ramsaroop L, Allopi L, Moodley J and Satyapal KS Duplicate gallbladder: An unusual case report. Surg Radiol Anat 28: 654-657, 2006.

13. Joliat GR, Shubert CR and Farley DR: Isolated congenital agenesis of the gallbladder and cystic duct: Report of a case. J Surg Educ 70: 117-120, 2013.

14. Singh B, Moodley J, Haffejee AA and Rajaruthnam P: Laparoscopic diagnosis of gallbladder agenesis. Surg Laparosc Endosc 7: 129-132, 1997.

15. Smadi SI, Naasan WA, Alkaabneh and Haddad JS: Agenesis of Gall Bladder. A Case Report. J Res Med Sci 12: 63-65, 2005.

16. Fiaschetti V, Calabrese G, Viarani S, Bazzocchi G and Simonetti G: Gallbladder agenesis and cystic duct absence in an adult patient diagnosed by magnetic resonance cholangiography: Report of a case and review of the literature. Case Rep Med 2009: 674768, 2009. 